\title{
DYNAMICAL MECHANISMS FOR TRANSFERRING COMETS IN AND OUT OF THE OORT CLOUD
}

\author{
M.J. Valtonen and J.Q. Zheng \\ Tuorla Observatory, University of Turku, 21500 Piikkio, Finland
}

\begin{abstract}
We study scenarios where comets are not original members of the solar System but have been acquired from the surrounding medium through dynamical evolution. This leads to estimates of the present day number density of comets in the interstellar medium which are not in contradiction with observat ional upper 1 imits. We al so consider the dynamical transfer of Oort Cloud comets into short period comets. The process is very sensitive to the inclination of the comet or bit which leads to strong bias in favour of low inclinations in short comet or bits.
\end{abstract}

\section{INTRODUCT ION}

Comets are in many ways odd members of the solar system. The majority of the comets, forming the so called Oort Cloud, are very weakly bound to the Sun. Also their orbits are randoml y inclined to the plane of the planetary system in contrast to the smaller number of strongly bound short period comets which follow mostly low inclination orbits. This poses a problem if one considers the short period comets as captured members of the Oort Cloud (Duncan, Quinn and Tremaine 1988). Al so there are questions about the adequancy of the capture rate (Joss 1973). The structure and origin of the Oort Cloud, as well as its ability to survive through the age of the solar System have been under discussion in recent years (Eailey et al. 1986, Stagg and Bailey 1989).

In this paper we summarize several dynamical studies which may shed 1 ight on the above cometary problems. We consider the origin of the Oort Cloud, its ability to survive 
stellar perturbations and the capture of short period comets from the Oort cloud.

\section{CAPTURE OF OORT CLOUD COMETS}

The extreme looseness of the clouds of comets which surrounds the solar system gives a clue to the origin of the system. If the comets were born with the rest of the solar system we might expect a disk-1ike distribution of comets somewhere at the periphery of the planetary system (Kuiper 1951). This kind of disk would be a natural source of short period comets (Fernandez 1980) but it is unclear how the orbits of the Oort Cloud comets could evolve from a kuiper disk. It may be attractive to consider the opposite trend where the comets become more strongly bound when time goes on. Initially comets are captured from the interstellar medium and form an oort Cloud. Stellar perturbations then deviate some of orbits to the inner solar system and a group of short period comets is formed.

The starting point of our scenario is a star cluster where the Sun is one of the newl y formed stars. The cluster may have several tens of other stars of different masses, and in addition to that, a swarm of comets as a left over from star formation (McCrea 1975, Donn 1976). We then follow what happens to the comets during the dynamical evolution of the star cluster. In particular, we are interested in the number of comets which attach themselves to a typical star such as the Sun, and the number of comets which end up as free members of interstellar medium.

Our calculations, reported in more detail by Zheng, Val tonen and Valtaoja (1991) and Valtonen and Zheng (1991), show that typically an Oort Cloud of $10^{12}$ comets (Oort 1950, Weissman 1982) is attached to a single, escaping star if the cluster has about $10^{1.6}$ comets initially. Most of these comets are dispersed in the interstellar space. If the process is common and occurs in all star clusters, a comet mass density of about $10^{-3}$ solar mass per cubic parsec arises in the Galaxy. The upper limit derived from not having ever observed a clear$1 y$ interstellar comet comes to about the same number (Sekanina 1976). Thus acquiring of the Oort Cloud by dynamical processes in a star cluster is at least possible. Such a large comet density would have important effects in the evolution of the Galaxy (Bailey 1988). The structure of the Oort Cloud formed by this process is not unlike the cloud deduced originally by Oort (1950), kut does not include the unobservable extension of the cloud inward ("inner Oort Cloud") which is often discussed (Bailey 1986).

\section{STABIL ITY OF THE DORT CLOUD OF COMETS}

The survival of the Oort Cloud of comets has been studied by several authors, see e.g. Bailey (1983). Zheng (1989) cal- 
culates the cumulative effect of 23000 stellar perturbations on comet orbits of different kinds. In the simulation the perihelion distances of the passing stars are randomly distributed between 0 and 1 pc, the orbits are randoml y oriented and the masses of the perturbing stars are randomly distributed between 0.8 and 1.2 solar mass. During the stellar encounter, the comet and the sun suffer changes in velocity given by

$$
\begin{aligned}
& \vec{\Delta}_{c}=(K / D) *(\vec{D} / D) \\
& \vec{V}_{0}=\left(K / D_{0}\right) *\left(\vec{D}_{0} / D_{0}\right) .
\end{aligned}
$$

and the cometary velocity relative to the Sun gains an increment

$$
\Delta \vec{V}=\Delta \vec{V}_{c}-\Delta \vec{v}_{0}
$$

where $K=2 G M / V$, (GM is gravitational mass of the passing stars, $\vec{V}$ is encounter velocity), and $\vec{D}$ is the vector of the closest distance between the comet and the star, $\vec{D}_{0}$ being that between the Sun and the star.

It is found that only about $3-9 \%$ of the Oort cloud survive through the age of the Solar System if the aphelion distance of the comets is $40000 \mathrm{AU}$. At the aphelion distance of $10000 \mathrm{AU}$ it is found that $26-80 \%$ of the comets survive, depending on the eccentricity of the orbit. Therefore we must assume that the present day Dort Cloud is only a remnant of a more populous initial Oort Cloud which had many $t$ imes more comets than the Oort cloud of today.

While many of the Oort cloud comets leave the Solar System, others enter the planetary region. Figure 1 shows the fraction of comets which escape, enter the planetary region or survive at the aphelion distance of $Q=40000 \mathrm{AU}$, when $t$ he perihelion distance is varied from $q=40 \mathrm{AU}$ to $q=40000 \mathrm{AU}$.

\section{CAPTURE JF SHORT PERIOD COMETS}

It has been generally assumed that short period comets are captured members of the Dort cloud comets. However, in recent years there has been a debate whether a random distribution of inclinations such as is found in the oort cloud could develop into the low inclination distribution of the short period comets (Stagg and Bailey 1989). In our numerical studies of the capture probability as a function of inclination we have found about $2: 1$ excess of zero inclination captures over the retrograde orbit captures (Valtonen and Innanen 1982). This may be compared with the observed distribution of inclinations of short period comets which show about $7: 1$ excess of direct (cosi $>0.8$ ) orbits over retrograde 


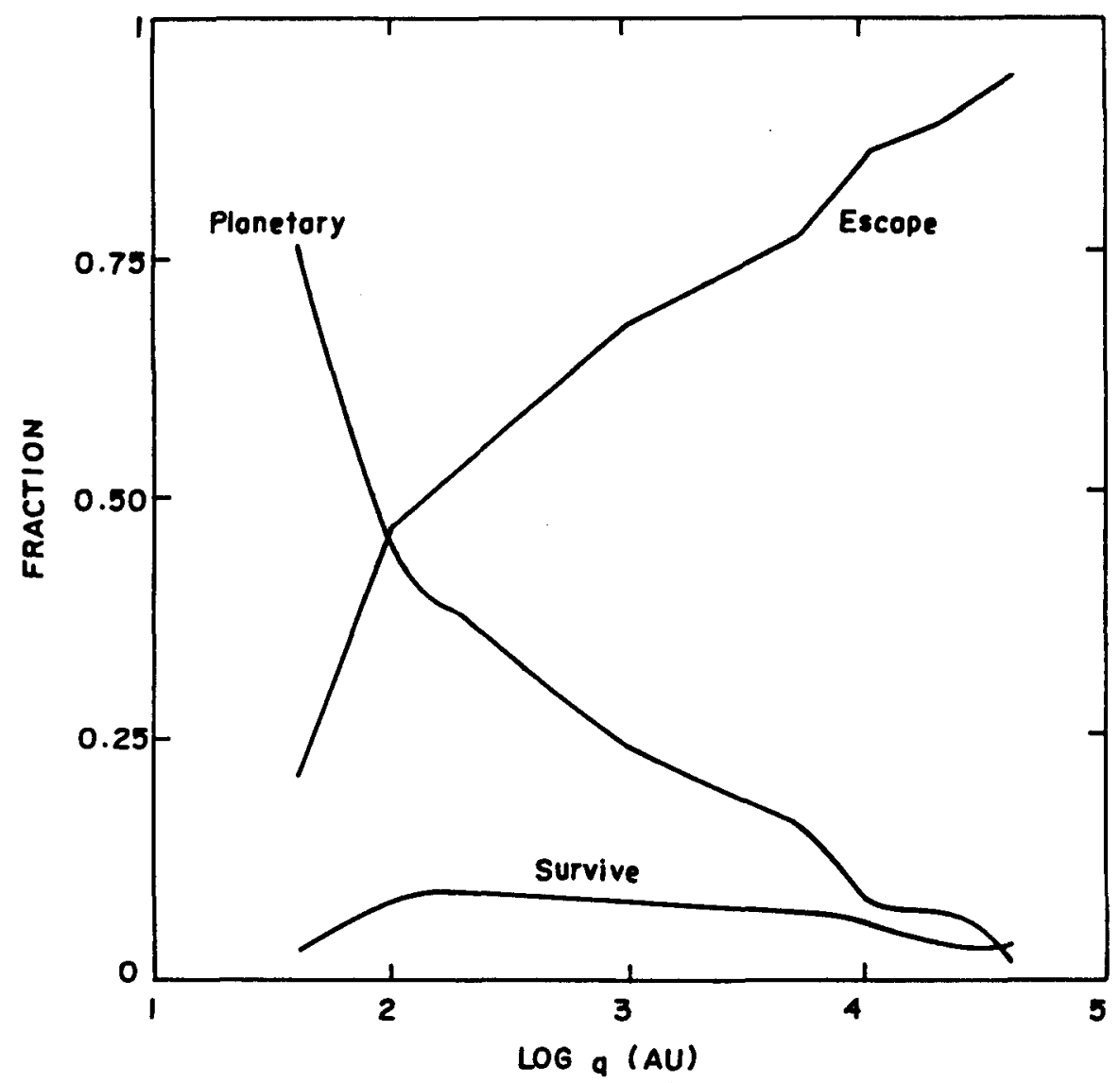

Figure 1: The final states of the Oort Cloud at aphelion distance $Q=40000$ AU at different perihelion distances q.

orbits (comets with aphelia > $10 \mathrm{AU}$; Stagg and Bailey 1989). If the comet capture proceeds onl y through the assistance of a single planet, then indeed the observed inclination distribution is definitely too strongly biased towards low inclina$t$ ions. However, if the comet is passed from the influence of one planet to the one lower to it and so on, then the above probabilities should be raised to power $\mathrm{N}$ of $\mathrm{t}$ he number of planets involved. For example, $N=3$ leads us to expect an $8: 1$ bias in favour of direct orbits. 


\section{CONCLUSIONS}

We have outlined a picture of the origin of both short period and long period comets starting from an interstellar population of comets in a star cluster in which the Sun was born. First the Sun accumulates a cloud of loosely bound comets, many of which are subsequently lost to the interstellar space due to stellar perturbations. Some comets are diverted to the inner Solar System and are captured via planetary influences. These form a cloud of short period comets with primarily low inclination orbits.

The primary test of this picture is to find a true interstellar comet, a member of the Galactic population which must necessarily arise during the process of break-up of a cluster of stars and comets. Other tests include more detailed studies of capture of short period comets from the Dort cloud, including the influence of several outer planets.

\section{REFERENCES}

[1] Bailey, M.E. (1983), Monthly Notices Roy. Astr. Soc.,204, 603 .

[2] Bailey, M.E. (1986), Monthly Notices Roy. Astron. Soc., 218,1 .

[3] Bailey, M.E. (1988), In Dust in the Universe (M.E.Biley and D.A. Williams, Eds.), p.113.

[4] Bailey, M.E., Clube, S.V.M. and Napier, W.M.(1986),Vistas A stron., 29,53.

[5] Donn, B. (1976), In The Study of Comets (B.Donn et al., Eds.), p.633.

[6] Duncan, M., Quinn, T. and Tremaine, S. (1988), Astrophys J ., 328, L69.

[7] Fernandez, J.A. (1980) Monthly Notices Roy. Astron.Soc., 192,481 .

[8] Joss, P.C. (1973), Ast ron. Astrophys. , 25, 271.

[9] Kuiper, G.P. (1951), In Astrophysics (J.A.Hynek, Ed.), p.357.

[10] McCrea, W.H. (1975), The Observatory, 95, 239.

[11] Oort, J.H. (1950), Bul1.Astron. Inst. Netherl ands,11, 91 .

[12] Sekanina, Z. (1976) Icarus, 27,123.

[13] Stagg, C.R. and Bailey, M.E. (1989), Monthly Notices Roy. A st ron. Soc., 241, 507 .

[14] Valtonen, M.J. and Innanen, K.A. (1982), Astrophys J.255, 307 .

[15] Valtonen, M.J. and Zheng, J.-Q. (1991), In Proceedings of the Nordic-Baltic Astronomy Meeting in Uppsala (C. -I. Lagerqvist et al., Eds.), in press.

[16] Weissman, P.R.(1982), In Comets (L.L. Wilkening, Ed.), p.637,

[17] Zheng, J . -Q. (1989), Acta Astron. Sinica 30,126.

[18] Zheng, J . $-Q$, Valtonen, M.J. and Valtaoja,L. (1991), Celestial Mechanics, in press. 